\title{
BUILDING THE EVIDENCE BASE TO ADDRESS HEALTH INEQUALITIES
}

\section{Helen Catchatoor}

Health Inequalities Research Collaboration Secretariat

The Australian Health Inequalities Research Collaboration (HIRC) was established by the Commonwealth Government in 1999. In the July 2001 issue of the NSW Public Health Bulletin (Volume 12, Number 7), Dixon and Sibthorpe, from the National Centre for Epidemiology and Population Health, which previously housed the HIRC Secretariat, reviewed the work of HIRC in its first 18 months. Here we provide an update of its activities.

HIRC is designed to assist research workers who are applying their energies to improving our understanding of health inequalities, what causes them, and how they may be ameliorated. This support is by way of clarifying research priorities, supporting networking by researchers, and advocating for more funding support for such research. HIRC is especially keen to foster research that tests interventions on the basis of sound theory.

Some interventions can appear relatively simple, although they may have an underlying complexity. For example, adding iodine or other trace elements to the diet of mothers and children who are deficient can save lives and prevent mental sub-normality. Culturally-appropriate interventions can also achieve health gain, such as the Strong Women, Strong Babies, Strong Culture project in the Northern Territory, involving antenatal care for indigenous women. ${ }^{1}$ This project achieved a 47 per cent reduction in low birth-weight, from 20.3 per cent to 11.5 per cent); and a 55 per cent reduction in the pre-term birth rate, from 21 per cent to 9.5 per cent. ${ }^{1}$

To make such interventions sustainable involves social and political commitment. In other cases, interventions are needed that take account of economic and environmental factors that are the root causes of health inequality. $^{2}$

HIRC's contribution to building capacity in relation to health inequalities research and development is articulated in its Strategic Action Plan 2001-2003. The key objectives of this plan are to:

- increase the national focus on reducing health inequalities;

- build national capacity and support for research and development in health inequalities;

- establish close collaboration among researchers, practitioners, and policy developers;

- promote the uptake of research findings in policy, practice, and evaluation.

Functioning as an institute without walls, HIRC is building virtual networks in a few key subject areas that the evidence suggests are instrumental to improving health and reducing health inequalities. These areas, and their coordinating teams, are:
- Children, Youth and Families - coordinated by Jan Nicholson, School of Public Health Research, Queensland University of Technology; Elizabeth Waters, Murdoch Children's Research Institute; and Graham Vimpani, University of Newcastle;

- Primary Health Care-coordinated by Elizabeth Harris, Centre for Health Equity Research, Training and Evaluation; John Furler, Department of General Practice, University of Melbourne; and Julie McDonald, network coordinator and private consultant;

- Sustainable Communities - coordinated by Pierre Horwitz, Edith Cowan University.

The three HIRC networks will be responsible for:

- establishing and maintaining a comprehensive and viable research network, and addressing rural health and the health of indigenous Australians;

- providing expert advice on priority research topics, questions, and related maters. This includes advice on the evidence for effective interventions;

- facilitating communication and collaboration between network members and other individuals and groups;

- undertaking activities to build capacity in research concerning health inequalities as it applies to the subject areas.

The HIRC will be judged against the following indicators:

- improved investment in health inequalities research by government and non-government organisations;

- research funding bodies accounting for what they spend on health inequalities;

- increased efforts at all levels to intervene to reduce health inequalities, as described in the published and unpublished literature;

- acknowledgement from indigenous Australian health organisations that HIRC has contributed to the effort to reduce health inequalities;

- effective network relationships between researchers, practitioners, and policy makers, as indicated by the activities of the HIRC-sponsored networks.

Researchers and others interested in the subject areas covered by the three HIRC networks are welcome to contact the network coordinators. For general information about HIRC, and how to contact the network coordinators, contact Helen Catchatoor, HIRC Secretariat by email at helen.catchatoor@health.gov.au or visit the HIRC Web site at www.hirc.health.gov.au.

\section{REFERENCES}

1. National Public Health Partnership online at http:// hna.ffh.vic.gov.au/nphp/key/key26/key26.htm.

2. Horton R. Public health: a neglected counter-terrorist measure. Lancet 358: October 6, 2001: 1112-13. 罳 